\title{
Polarization-Correlated Photon Pairs from a Single Quantum Dot
}

\author{
Charles Santori, : , David Fattal, Matthew Pelton, Glenn S. Solomon, 周 and Yoshihisa Yamamotof \\ Quantum Entanglement Project, ICORP, JST, E.L. Ginzton Laboratory, \\ Stanford University, Stanford, California 94305
}

(Dated: October 24, 2018)

\begin{abstract}
Polarization correlation in a linear basis, but not entanglement, is observed between the biexciton and single-exciton photons emitted by a single InAs quantum dot in a two-photon cascade. The results are well described quantitatively by a probabilistic model that includes two decay paths for a biexciton through a non-degenerate pair of one-exciton states, with the polarization of the emitted photons depending on the decay path. The results show that spin non-degeneracy due to quantumdot asymmetry is a significant obstacle to the realization of an entangled-photon generation device.
\end{abstract}

PACS numbers: 78.67.Hc, 42.50.Ar, 78.55.Cr

New nonclassical light sources are needed for recently proposed optical implementations of quantum cryptography [1] and quantum computation [2]. Single semiconductor quantum dots [3] are attractive as nonclassical light sources because they have engineered properties, do not suffer from photobleaching effects, and can be integrated into larger structures to make monolithic devices. Quantum dots have already shown potential as single-photon sources [4, 5, 6], but they can also generate sequences of photons in a radiative cascade [7, \#]. In such a cascade, each photon has a unique wavelength, and the photons may also have correlated, or even entangled [9] polarizations.

In the two-photon cascade, a biexciton singlet state (two electrons and two holes, 2X) decays to one of two optically active single-exciton states (one electron and one hole, 1X) by emitting one photon, and then to the empty-dot state by emitting a second photon. In theory, the polarization properties of photon pairs emitted through these two decay paths result entirely from properties of the optically-active $1 \mathrm{X}$ doublet 110, 11, 12. For a symmetric quantum dot, the two $1 \mathrm{X}$ states are degenerate, and the two decay paths become "indistinguishable," ideally producing polarization-entangled photons [9]. For an asymmetric quantum dot, the $1 \mathrm{X}$ doublet is split through the electron-hole exchange interaction into states that couple to photons having orthogonal linear polarizations 13, 14, 15. If this splitting is much larger than the radiative linewidth, then the two paths become "distinguishable," with one decay path producing two horizontally-polarized photons and the other producing two vertically polarized photons, for example. In this case, polarization correlation is expected only in a single, preferred basis. Some additional factors are also important, such as spin flip [16] and decoherence processes 17, 18, 19 that randomize the intermediate $1 \mathrm{X}$ state, and valence-band mixing [20].

In this article, we present an experimental study of the polarization correlation properties of photon pairs emitted through biexciton decay in a single InAs quantum dot. While temporal correlations between $1 \mathrm{X}$ and $2 \mathrm{X}$ photons have previously been seen 21], polarization correlation has not yet been reported, to our knowledge, although a lack of such correlation has been mentioned elsewhere 22. For our sample, we observe a strong polarization correlation in a linear polarization basis, verifying the theoretical picture described above. However, we do not observe entanglement, suggesting that quantum-dot asymmetry is an obstacle to realizing an entangled photon source.

A sample was fabricated containing self-assembled InAs quantum dots [3] grown by molecular-beam epitaxy on a (001) GaAs substrate, capped by $75 \mathrm{~nm}$ of GaAs. A high growth temperature increased intermixing between the InAs and surrounding GaAs, shortening the quantum-dot emission wavelengths. Mesas about $120 \mathrm{~nm}$ tall, $200 \mathrm{~nm}$ wide, and spaced $50 \mu \mathrm{m}$ apart were fabricated by electron-beam lithography and dry etching. The dots are sparse enough $\left(11 \mu \mathrm{m}^{-2}\right)$ that the smallest mesas contain, on average, fewer than one dot.

The setup used to characterize the quantum-dot emission is shown in Fig. 11. The sample was cooled to 3$5 \mathrm{~K}$ in a cryostat. Single mesas were excited above the GaAs bandgap $(710 \mathrm{~nm})$ by horizontally polarized, $3 \mathrm{ps}$ Ti-Sapphire laser pulses every $13 \mathrm{~ns}$, using a beam incident $54^{\circ}$ from normal and focussed to a $20 \mu \mathrm{m}$ spot size on the sample surface. The emission from the dot was collected by an $\mathrm{NA}=0.5$ aspheric lens, spectrally filtered to remove laser scatter, imaged onto a pinhole that selects emission from a $7 \mu \mathrm{m}$-wide region of the sample, and sent to a Hanbury Brown and Twiss-type (HBT) correlation setup. The HBT setup begins with a nonpolarizing beamsplitter, followed by retarders to correct for the polarization-dependent phase shifts caused by this beamsplitter. Each arm then includes a rotatable half-wave plate to select the measurement polarization, a horizontal polarizer, a small monochromator $(0.35 \mathrm{~nm}$ resolution), and an avalanche-photodiode photon counter (EG\&G SPCM) having about $200 \mathrm{~s}^{-1}$ dark counts. The electrical pulses from the photon counters served as "start" $\left(t_{1}\right)$ and "stop" $\left(t_{2}\right)$ triggers for a time-to-amplitude converter (TAC), whose output was converted to a histogram by a 


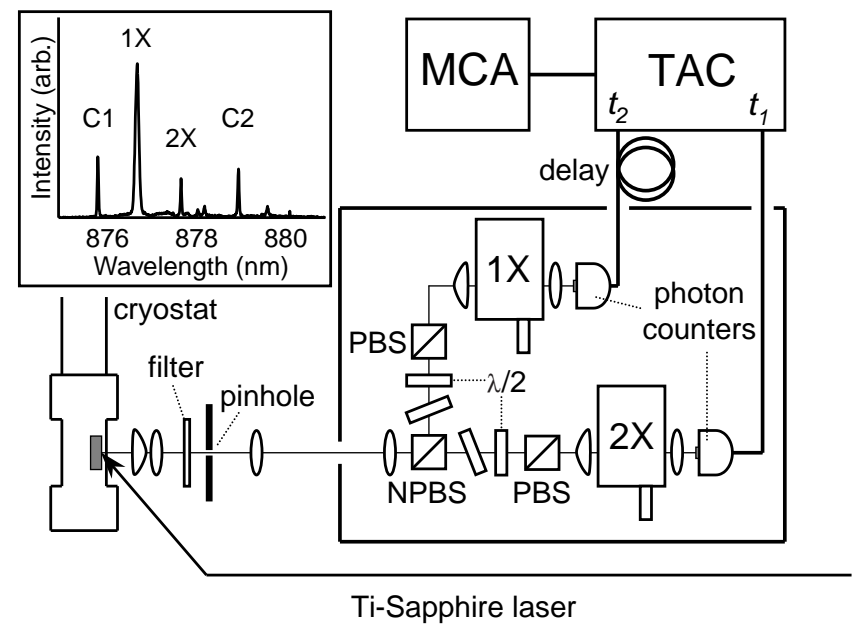

FIG. 1: Photon correlation setup: A modelocked TiSapphire laser excites the sample inside of a cryostat. The collected emission is split into two arms by a nonpolarizing beamsplitter (NPBS). The two arms count $1 \mathrm{X}$ and $2 \mathrm{X}$ photons, with rotatable half-wave plates $(\lambda / 2)$ followed by polarizers (PBS) determining the measurement polarizations. An electronic system (delay, TAC, MCA) generates the photon correlation histogram. Inset: single-dot emission spectrum under CW, $650 \mathrm{~nm}$ excitation, showing the single-exciton (1X), biexciton (2X), and charged-exciton (C1, C2) lines.

multi-channel analyzer (MCA) card in a computer. The resulting histogram of time intervals $\tau=t_{2}-t_{1}$ is equivalent to a measurement of the photon correlation function, since the collection efficiency is extremely low. The "stop" signal was delayed by $100 \mathrm{~ns}$ to allow both negative and positive values of $\tau$ in the correlation histogram.

A photoluminescence spectrum of the dot chosen for this study under continuous-wave $(\mathrm{CW})$, above-band excitation $(650 \mathrm{~nm})$ is shown in the inset of Fig. 1. The lines labeled $1 \mathrm{X}$ and $2 \mathrm{X}$ are identified as one-exciton and biexciton emission, respectively, while the lines labeled $\mathrm{C} 1$ and $\mathrm{C} 2$ are identified as charged-exciton [23] emission. For all of the correlation measurements to be presented, the "start" counter was tuned to the $2 \mathrm{X}$ line, and the "stop" counter was tuned to the $1 \mathrm{X}$ line.

This quantum dot has a large polarization anisotropy. For convenience, we designate " $\mathrm{H}$ " to be a linear polarization rotated $18^{\circ}$ from the horizontal lab axis (chosen to maximize the observed polarization correlations), and "V" as the orthogonal polarization. The horizontal lab axis is aligned with one of the two GaAs cleave directions, (110) or (1-10). The photon count rate for $\mathrm{H}$ is nearly double that for $\mathrm{V}$. The normalized Stokes vectors of the $2 \mathrm{X}$ and $1 \mathrm{X}$ lines are $\mathbf{S}_{2 X}=(0.34,-0.09,-0.08)$ and $\mathbf{S}_{1 X}=(0.28,-0.12,0.04)$, respectively, where the three components are the intensity visibilities in the $\mathrm{H} / \mathrm{V}$, $+45^{\circ} /-45^{\circ}$, and circular bases, respectively. Most dots on this sample have polarization anisotropy, though the direction of the Stokes vector varies. Such anisotropy

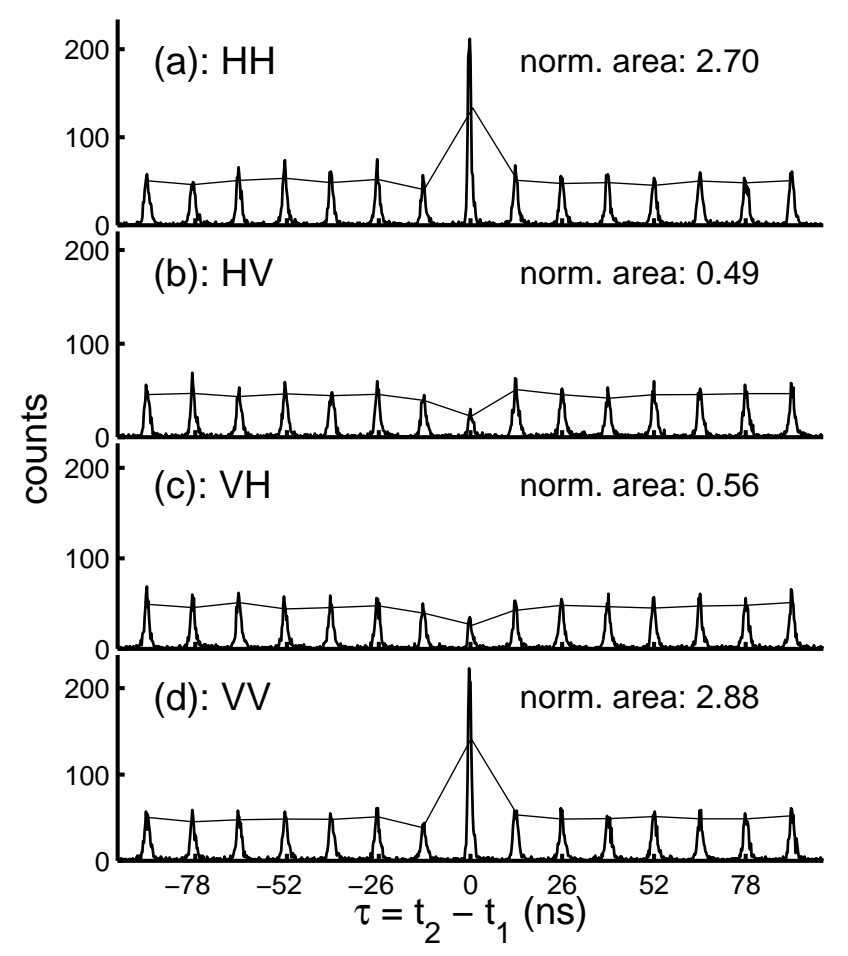

FIG. 2: Photon correlation histograms between the $2 \mathrm{X}$ and 1X emission lines for four polarization combinations: (a) $\mathrm{HH}$, (b) $\mathrm{HV}$, (c) VH, and (d) VV, where the first and second letters refer to the $2 \mathrm{X}$ and $1 \mathrm{X}$ polarizations, respectively. $\mathrm{H}$ is a linear polarization rotated $18^{\circ}$ from lab horizontal, and $\mathrm{V} \perp \mathrm{H}$. The central peak at $\tau=0$ results from $2 \mathrm{X}-1 \mathrm{X}$ coincidences, and its area, normalized relative to the side peak average, is indicated. The solid lines indicate relative peak areas. The large $\mathrm{HH}$ and $\mathrm{VV}$ central peak areas and small $\mathrm{HV}$ and $\mathrm{VH}$ areas demonstrate polarization correlation.

has been reported elsewhere [24, 25], and is related to the asymmetry of the quantum dot or its environment.

Photon correlation histograms for four special polarization combinations are shown in Fig. 2. The histograms display a series of peaks, separated by the laser repetition period. Counts in the central peak at $\tau=0$ occur when both a $2 \mathrm{X}$ photon and a $1 \mathrm{X}$ photon are detected following the same laser pulse, and its area is proportional to the $2 \mathrm{X}-1 \mathrm{X}$ coincidence rate. Counts in the side peaks occur when two photons are detected that resulted from different laser pulses. The side peaks far from $\tau=0$ provide an uncorrelated normalization standard, with areas proportional to the product of the $2 \mathrm{X}$ and $1 \mathrm{X}$ count rates. The integration times were chosen to yield approximately the same side peak area for each histogram. A rise of the side peaks near $\tau=0$ as reported in [A] does not appear here, because the excitation energy here is above the GaAs bandgap.

It is clear from Fig. 2 that, in the chosen measurement basis, a large degree of polarization correlation exists between the $2 \mathrm{X}$ and $1 \mathrm{X}$ photons. The central peak 


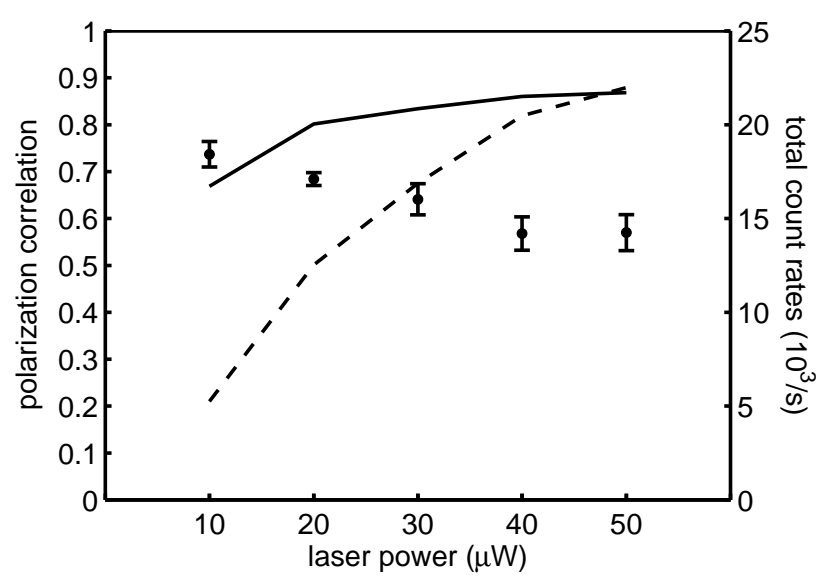

FIG. 3: Left axis: measured polarization correlation function $\chi_{H V}$, as defined in the text (Eq. 1), versus pump laser power (points). The error bars include only Poisson photon number statistics. Right axis: total count rates (summed over orthogonal polarizations) for $1 \mathrm{X}$ emission (solid line) and $2 \mathrm{X}$ emission (dashed line).

appears much larger relative to the side peaks for the $\mathrm{H}_{2 \mathrm{X}} / \mathrm{H}_{1 \mathrm{X}}$ and $\mathrm{V}_{2 \mathrm{X}} / \mathrm{V}_{1 \mathrm{X}}$ cases $(\mathrm{a}, \mathrm{d})$ than for the $\mathrm{H}_{2 \mathrm{X}} / \mathrm{V}_{1 \mathrm{X}}$ and $\mathrm{V}_{2 \mathrm{X}} / \mathrm{H}_{1 \mathrm{X}}$ cases $(\mathrm{b}, \mathrm{c})$. We choose to quantify the degree of correlation by the function

$$
\chi_{H V}=\frac{\sqrt{C_{\mathrm{HH}} C_{\mathrm{VV}}}-\sqrt{C_{\mathrm{HV}} C_{\mathrm{VH}}}}{\sqrt{C_{\mathrm{HH}} C_{\mathrm{VV}}}+\sqrt{C_{\mathrm{HV}} C_{\mathrm{VH}}}},
$$

where $C_{\alpha \beta}$ is the coincidence rate for $2 \mathrm{X}$ and $1 \mathrm{X}$ measurement polarizations of $\alpha$ and $\beta$, respectively. This function yields values of $+1,0$ and -1 for the cases of perfect polarization correlation, independent polarizations, and perfect polarization anticorrelation, respectively, and is simply related to the polarization-flip probability $\epsilon$ in the model described below. The data shown in Fig. 2 were acquired with $20 \mu \mathrm{W}$ excitation power, producing a correlation of $\chi_{H V}=0.684$. The measured value of $\chi_{H V}$ for a range of excitation powers is plotted in Fig. 3. The $2 \mathrm{X}$ and $1 \mathrm{X}$ count rates are also shown. A large degree of correlation occurs even when the $2 \mathrm{X}$ and $1 \mathrm{X}$ count rates are close to saturation.

While a strong polarization correlation is seen in the $\mathrm{H} / \mathrm{V}$ basis, negligible correlation is seen in the $+45^{\circ} /-45^{\circ}$ basis $\left(\chi_{+45,-45}=0.055\right)$, suggesting that the photon pairs have negligible entanglement. To obtain the entire two-photon density matrix, we followed the procedure outlined in [26]. The density matrix can be determined from the coincidence rates measured with the following $2 \mathrm{X}-1 \mathrm{X}$ polarization combinations: $\mathrm{HH}, \mathrm{HV}$, VH, VV, HD, HL, DH, RH, DD, RD, RL, DR, DV, RV, $\mathrm{VD}$, and VL, where the first and second letters refer to the $2 \mathrm{X}$ and $1 \mathrm{X}$ measurement polarizations, respectively, $\mathrm{D}$ refers to $+45^{\circ}$, and $\mathrm{R}$ and $\mathrm{L}$ are the orthogonal circular polarizations. For measurement combinations including a circular polarization, a single quarter-wave plate was
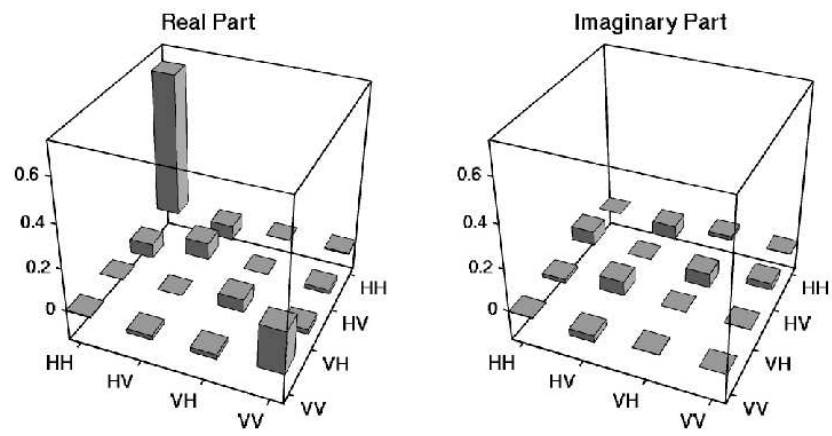

FIG. 4: Graphical representation of the two-photon polarization density matrix describing the $2 \mathrm{X}$ and $1 \mathrm{X}$ collected photons under $20 \mu W$ excitation power.

inserted after the collection lens. To minimize the effect of sample position drift, a significant error source, we calculated the coincidence rate from the ratio of the central correlation peak area to the more distant side peak areas:

$$
D_{\alpha \beta}=\frac{C_{\alpha \beta}(0)}{\bar{C}_{\alpha \beta}\left(\tau_{n}\right)} \frac{1+\mathbf{S}_{2 X} \cdot \mathbf{M}_{2 X}}{2} \frac{1+\mathbf{S}_{1 X} \cdot \mathbf{M}_{1 X}}{2},
$$

where $D_{\alpha \beta}$ is the corrected coincidence rate for $2 \mathrm{X}$ and $1 \mathrm{X}$ polarizations $\alpha$ and $\beta$, respectively, $C_{\alpha \beta}(0)$ is the raw central peak area, $\bar{C}_{\alpha \beta}\left(\tau_{n}\right)$ is the mean area of the more distant side peaks, $\mathbf{S}_{2 X}$ and $\mathbf{S}_{1 X}$ are the $2 X$ and $1 \mathrm{X}$ normalized Stokes vectors, and $\mathbf{M}_{2 X}$ and $\mathbf{M}_{1 X}$ are the Stokes vectors of the $2 \mathrm{X}$ and $1 \mathrm{X}$ measurement polarizations. The effect of sample drift is largely canceled, since $C_{\alpha \beta}(0)$ and $\bar{C}_{\alpha \beta}\left(\tau_{n}\right)$ both depend on the square of the collection efficiency. The polarization dependence of $\bar{C}_{\alpha \beta}\left(\tau_{n}\right)$ is canceled by the last two terms.

The normalized density matrix obtained for a pump power of $20 \mu \mathrm{W}$ is shown in Fig. 4 . The relatively small off-diagonal elements show that little, if any, entanglement is present. This matrix can in fact be shown by the Peres criterion to be separable [27]. The on-diagonal components display the polarization correlation that appears in the $\mathrm{H} / \mathrm{V}$ basis. Their values are given in Table [.

To model our results, we consider a two-path decay process for the initial biexciton, as described in 14]. In one path, both photons are emitted with $\pi_{x}$ polarization (detected as $\mathrm{H}$ ), while in the other path, both are $\pi_{y}$ (detected as $\mathrm{V}$ ). We assume that the two paths occur with equal probability. We also include a probability $\epsilon$ that the two photons have opposite polarizations. This takes into account both spin flipping in the oneexciton state, and the possibility of non-ideal selection rules. The coincidence probabilities are then $C_{\alpha \alpha}=$ $\eta_{\alpha, 2 X} \eta_{\alpha, 1 X}(1-\epsilon) / 2$ and $C_{\alpha \beta}=\eta_{\alpha, 2 X} \eta_{\beta, 1 X} \epsilon / 2$, where $\alpha$ represents either $\mathrm{H}$ or $\mathrm{V}$ polarization, $\beta \perp \alpha$, and $\eta_{\alpha, 2 X}$ and $\eta_{\alpha, 1 X}$ are the $2 \mathrm{X}$ and $1 \mathrm{X}$ polarization-dependent collection efficiencies. To compare this model to the data, we calculate the collection efficiency ratios from the measured Stokes vectors, obtaining $\eta_{V, 2 X} / \eta_{H, 2 X}=0.494$ 
TABLE I: On-diagonal elements of the two-photon polarization density matrix, measured and predicted.

\begin{tabular}{lcccc}
\hline \hline & $\rho_{H H, H H}$ & $\rho_{H V, H V}$ & $\rho_{V H, V H}$ & $\rho_{V V, V V}$ \\
Measured & 0.669 & 0.078 & 0.059 & 0.194 \\
Model & 0.678 & 0.071 & 0.063 & 0.188 \\
\hline \hline
\end{tabular}

and $\eta_{V, 1 X} / \eta_{H, 1 X}=0.560$. To estimate $\epsilon$, we note that $\epsilon=\left(1-\chi_{H V}\right) / 2=0.158$, where $\chi_{H V}$ is defined in Eq. 1. The on-diagonal density matrix elements predicted using these parameters are given in Table [], and are in close agreement with the measured values. From this model, we can infer that the $1 \mathrm{X}$ polarization flip time $T_{1}$ is at least $\tau_{\text {rad }}(1-2 \epsilon) / \epsilon=2.2 \mathrm{~ns}$, where $\tau_{\text {rad }}=0.5 \mathrm{~ns}$ is the $1 \mathrm{X}$ recombination lifetime.

The large difference between $\rho_{H H, H H}$ and $\rho_{V V, V V}$ is related to the unequal detection rates of $\mathrm{H}$ and $\mathrm{V}$ photons. In the model above, we assume that this is due to different collection efficiencies for $\pi_{x}$ and $\pi_{y}$ photons. One might alternatively assume preferential decay through the $\pi_{x}$ path, but this explanation cannot simultaneously predict the measured two-photon density matrix and the measured single-photon Stokes vectors. The collection efficiencies for $\pi_{x}$ and $\pi_{y}$ must be different, perhaps due to the different angular dipole radiation patterns for $\pi_{x}$ and $\pi_{y}$ photons [20].

The fact that we see polarization correlation in only one linear basis and not entanglement suggests that quantum-dot asymmetry is a dominant effect. Neglecting spin relaxation, we calculate that the reduced offdiagonal density matrix element $\rho_{H H, V V}$ produced by an ideal two-photon cascade is $0.5 /\left(1+i \Delta \omega \tau_{\text {rad }}\right)$, where $\Delta \omega$ is the frequency splitting of the $1 \mathrm{X}$ state, and $\tau_{\text {rad }}$ is the $1 \mathrm{X}$ radiative lifetime. When $\Delta \omega \tau_{\text {rad }} \gg 1, \rho_{H H, V V}$ vanishes, and the entanglement disappears. This is expected, since the two decay paths can then be distinguished by the energies of the emitted photons. We infer from the fact that we do not see significant entanglement that $\hbar \Delta \omega \gg 1.3 \mu \mathrm{eV}$, using $\tau_{\text {rad }}=0.5 \mathrm{~ns}$, but we also know that $\hbar \Delta \omega<50 \mu \mathrm{eV}$, since we cannot resolve the polarization splitting spectrally. Since similar spin splittings have been reported for other material systems [19, 25, it appears that spin splitting will be a major obstacle to the realization of an entangled-photon device.

Several possible remedies might be used to reduce the ratio of the $1 \mathrm{X}$ energy splitting to the spontaneous emission rate. One could attempt to reduce $\Delta \omega$ by optimizing quantum-dot growth methods, and for this purpose a systematic study would be useful. It may also be possible to force the $1 \mathrm{X}$ states of an asymmetric quantum dot into degeneracy by applying an electric field or a strain to the sample. Spin relaxation would remain an issue, but a recent experimental result shows that the $1 \mathrm{X}$ spin decoherence time $T_{2}$ can be much longer than the radiative lifetime in CdSe quantum dots [19]. Alternatively, one could reduce $\tau_{\text {rad }}$, either by using larger quantum dots [28], or by placing the $1 \mathrm{X}$ state on resonance with a microcavity [29]. It has already been demonstrated that the spontaneous emission rates of quantum dots can be enhanced by a factor of up to 5 in pillar microcavities [30]. We hope that by one or more of these methods, a quantum-dot source of entangled photons may eventually be demonstrated.

The authors thank H. Kamada and O. Benson for valuable discussions. This work is partly supported by MURI DAAD19-00-1-0172 (UCLA). G.S.S. acknowledges support from DARPA, ARO and JST.

* Electronic address: chars@stanford.edu

$\dagger$ Also at Solid-State Photonics Laboratory, Stanford University.

¥ Also at NTT Basic Research Laboratories, Atsugishi, Kanagawa, Japan.

[1] For a review, see, N. Gisin, G. Ribordy, W. Tittel, and H. Zbinden, Rev. Mod. Phys. 74, 145 (2002).

[2] E. Knill, R. Laflamme, and G. J. Milburn, Nature (London) 409, 46 (2001).

[3] D. Bimberg, M. Grundmann, and N. Ledentsov, Quantum Dot Heterostructures (John Wiley \& Sons, Chichester, 1999).

[4] C. Santori, M. Pelton, G. Solomon, Y. Dale, and Y. Yamamoto, Phys. Rev. Lett. 86, 1502 (2001).

[5] P. Michler, A. Kiraz, C. Becher, W. V. Schoenfeld, P. M. Petroff, L. Zhang, E. Hu, and A. Imamoğlu, Science 290, 2282 (2000).

[6] V. Zwiller, H. Blom, P. Jonsson, N. Panev, S. Jeppesen, T. Tsegaye, E. Goobar, M.-E. Pistol, L. Samuelson, and G. Björk, Appl. Phys. Lett. 78, 2476 (2001).

[7] G. Bacher, R. Weigand, J. Seufert, V. D. Kulakovskii, N. A. Gippius, A. Forchel, K. Leonardi, and D. Hommel, Phys. Rev. Lett. 83, 4417 (1999).

[8] E. Dekel, D. V. Regelman, D. Gershoni, E. Ehrenfreund, W. V. Schoenfeld, and P. M. Petroff, Phys. Rev. B 62, 11038 (2000).

[9] O. Benson, C. Santori, M. Pelton, and Y. Yamamoto, Phys. Rev. Lett. 84, 2513 (2000).

[10] A. Kuther, M. Bayer, A. Forchel, A. Gorbunov, V. B. Timofeev, F. Schäfer, and J. P. Reithmaier, Phys. Rev. B 58, R7508 (1998).

[11] Y. Toda, S. Shinomori, K. Suzuki, and Y. Arakawa, Phys. Rev. B 58, R10147 (1998).

[12] M. Sugisaki, H. -W. Ren, S. V. Nair, K. Nishi, S. Sugou, T. Okuno, and Y. Masumoto, Phys. Rev. B 59, R5300 (1999).

[13] S. V. Gupalov, E. L. Ivchenko, and A. V. Kavokin, JETP 86, 388 (1998).

[14] V. D. Kulakovskii, G. Bacher, R. Weigand, T. Kümmell, A. Forchel, E. Borovitskaya, K. Leonardi, and D. Hommel, Phys. Rev. Lett 82, 1780 (1999).

[15] T. Takagahara, Phys. Rev. B 62, 16840 (2000).

[16] H. Kamada, H. Gotoh, H. Ando, J. Temmyo, and T. Tamamura, Phys. Rev. B 60. 5791 (1999).

[17] V. K. Kalevich, M. N. Tkachuk, P. Le Jeune, X. Marie, and T. Amand, Phys. Solid State 41, 789 (1999). 
[18] R. J. Epstein, D. T. Fuchs, W. V. Schoenfeld, P. M. Petroff, and D. D. Awschalom, Appl. Phys. Lett. 78, 733 (2001).

[19] T. Flissikowski, A. Hundt, M. Lowisch, M. Rabe, and F. Henneberger, Phys. Rev. Lett. 86, 3172 (2001).

[20] L. W. Wang, J. Kim, and A. Zunger, Phys. Rev. B 59, 5678 (1999).

[21] E. Moreau, I. Robert, L. Manin, V. Thierry-Mieg, J. M. Gérard, and I. Abram, Phys. Rev. Lett. 87, 183601 (2001).

[22] A. Kiraz, S. Fälth, C. Becher, B. Gayral, W. V. Schoenfeld, P. M. Petroff, L. Zhang, E. Hu, and A. Imamoğlu, Phys. Rev. B 65, 161303 (2002).

[23] J. J. Finley, P. W. Fry, A. D. Ashmore, A. Lemaître, A. I. Tartakovskii, R. Oulton, D. J. Mowbray, M. S. Skolnick, M. Hopkinson, P. D. Buckle, and P. A. Maksym,
Phys. Rev. B 63, 161305(R) (2001).

[24] Y. Nabetani, T. Ishikawa, S. Noda, and A. Sasaki, J. Appl. Phys. 76, 347 (1994).

[25] D. Gammon, E. S. Snow, B. V. Shanabrook, D. S. Katzer, and D. Park, Phys. Rev. Lett. 76, 3005 (1996).

[26] A. G. White, D. F. V. James, P. H. Eberhard, and P. G. Kwiat, Phys. Rev. Lett. 83, 3103 (1999).

[27] A. Peres, Phys. Rev. Lett. 77, 1413 (1996).

[28] J. Bellessa, V. Voliotis, R. Grousson, X. L. Wang, M. Ogura, and H. Matsuhata, Phys. Rev. B 58, 9933 (1998).

[29] J.- M. Gérard and B. Gayral, J. Lightwave Technol. 17, 2089 (1999).

[30] G. S. Solomon, M. Pelton, and Y. Yamamoto, Phys. Rev. Lett. 86, 3903 (2001). 